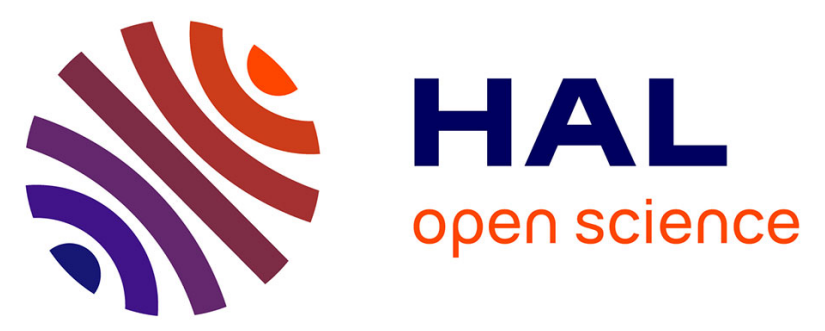

\title{
Global profiling of toxicologically relevant metabolites in urine: case study of reactive aldehydes
}

Emilien L. Jamin, Robin Costantino, Lö̈c Mervant, Jean-Francois Martin, Isabelle Jouanin, Florence Blas-Y-Estrada, Françoise Guéraud, Laurent Debrauwer

\section{To cite this version:}

Emilien L. Jamin, Robin Costantino, Loïc Mervant, Jean-Francois Martin, Isabelle Jouanin, et al.. Global profiling of toxicologically relevant metabolites in urine: case study of reactive aldehydes. Analytical Chemistry, 2020, 92 (2), pp.1746-1754. 10.1021/acs.analchem.9b03146 . hal-02622690

\section{HAL Id: hal-02622690 https://hal.inrae.fr/hal-02622690}

Submitted on 26 May 2020

HAL is a multi-disciplinary open access archive for the deposit and dissemination of scientific research documents, whether they are published or not. The documents may come from teaching and research institutions in France or abroad, or from public or private research centers.
L'archive ouverte pluridisciplinaire HAL, est destinée au dépôt et à la diffusion de documents scientifiques de niveau recherche, publiés ou non, émanant des établissements d'enseignement et de recherche français ou étrangers, des laboratoires publics ou privés. 


\section{analytiçal}

Article

Subscriber access provided by INRAE Institut National de Recherche pour l'Agriculture, l'Alimentation et l'Environnement

\section{Global profiling of toxicologically relevant metabolites in urine: case study of reactive aldehydes \\ Emilien L Jamin, Robin Costantino, Loïc Mervant, Jean-Francois Martin, Isabelle JOUANIN, Florence Blas-Y-Estrada, Françoise Guéraud, and Laurent Debrauwer}

Anal. Chem., Just Accepted Manuscript • DOI: 10.1021/acs.analchem.9b03146 • Publication Date (Web): 19 Dec 2019

Downloaded from pubs.acs.org on January 6, 2020

\section{Just Accepted}

"Just Accepted" manuscripts have been peer-reviewed and accepted for publication. They are posted online prior to technical editing, formatting for publication and author proofing. The American Chemical Society provides "Just Accepted" as a service to the research community to expedite the dissemination of scientific material as soon as possible after acceptance. "Just Accepted" manuscripts appear in full in PDF format accompanied by an HTML abstract. "Just Accepted" manuscripts have been fully peer reviewed, but should not be considered the official version of record. They are citable by the Digital Object Identifier (DOI®). "Just Accepted" is an optional service offered to authors. Therefore, the "Just Accepted" Web site may not include all articles that will be published in the journal. After a manuscript is technically edited and formatted, it will be removed from the "Just Accepted" Web site and published as an ASAP article. Note that technical editing may introduce minor changes to the manuscript text and/or graphics which could affect content, and all legal disclaimers and ethical guidelines that apply to the journal pertain. ACS cannot be held responsible for errors or consequences arising from the use of information contained in these "Just Accepted" manuscripts. 


\section{Global profiling of toxicologically relevant metabolites in urine: case study of}

\section{2 reactive aldehydes}

3

4

Emilien L. Jamin*o(1)(2), Robin Costantino*(1)(2), Loïc Mervant(1)(2), Jean-François Martin(1)(2), Isabelle Jouanin(1)(2), Florence Blas-y-Estrada(2), Françoise Guéraud*(2) and Laurent Debrauwer*(1)(2)

(1) Metatoul-AXIOM platform, National Infrastructure for Metabolomics and Fluxomics: MetaboHUB, Toxalim, INRAE, 31300 Toulouse, France

(2) Toxalim (Research Centre in Food Toxicology), Toulouse University, INRAE UMR 1331, ENVT, INPPurpan, UPS, 31300 Toulouse, France.

* Equal contribution

• Corresponding author: emilien.jamin@inra.fr

\section{Abstract}

Among the numerous unknown metabolites representative of our exposure, focusing on toxic compounds should provide more relevant data to link exposure and health. For that purpose, we developed and applied a global method using data independent acquisition (DIA) in mass spectrometry to profile specifically electrophilic compounds originating metabolites. These compounds are most of the time toxic, due to their chemical reactivity towards nucleophilic sites present in biomacromolecules. The main line of cellular defense against these electrophilic molecules is conjugation to glutathione, then metabolization into mercapturic acid conjugates (MACs). Interestingly, MACs display a characteristic neutral loss in MS/MS experiments, that makes possible to detect all the metabolites displaying this characteristic loss, thanks to the DIA mode, and therefore to highlight the corresponding reactive metabolites. As a proof of concept, our workflow was applied to the toxicological issue of the oxidation of dietary polyunsaturated fatty acids, leading in particular to the 
27 formation of toxic alkenals, which lead to MACs upon glutathione conjugation and metabolization. By

28 this way, dozens of MACs were detected and identified. Interestingly, multivariate statistical analyses

29 carried out only on extracted HRMS signals of MACs yield a better characterization of the studied

30 groups compared to results obtained from a classic untargeted metabolomics approach.

31

32

\section{TABLE OF CONTENTS GRAPHIC}

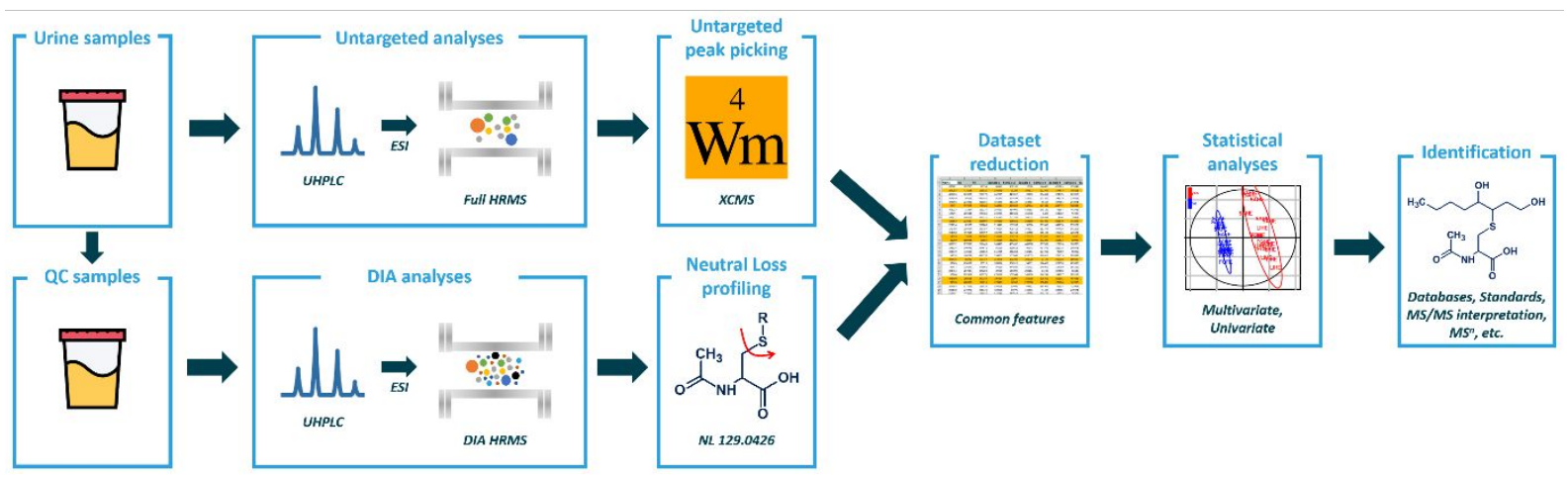




\section{INTRODUCTION}

Throughout their lives, humans are exposed to many substances, natural or not, in varying amounts depending on their environment, lifestyle or diet. In particular through diet, people are exposed to a wide variety of compounds mostly essential (e.g. nutrients, vitamins, etc...), but also, in a much lesser extent, to compounds with possible harmful effects such as pesticides, ${ }^{1}$ mycotoxins, ${ }^{2}$ or neo-formed compounds produced during the processing, cooking and digestion of food. ${ }^{3}$ Since the concept of exposomics has been introduced, ${ }^{4}$ new approaches aim at assessing this exposure in the most comprehensive way by the implementation of untargeted methods. ${ }^{5}$ However, in front of the number and complexity of these contaminants representing our chemical exposome, focusing on toxic compounds appears essential to estimate the impact of this exposure on health. Many of these toxicologically relevant compounds are toxic due to their electrophilicity, which makes them reactive towards nucleophilic sites of biomolecules such as DNA, RNA, proteins..., and lead to the formation of various adducts with potential harmful effects. Structure-activity relationships analyses carried out by Ashby and Tennant ${ }^{6}$ on the basis of carcinogenesis data from US-NTP showed that more than $80 \%$ of genotoxic agents are electrophilic compounds or compounds that are able to be bioactivated into electrophilic metabolites. In particular, meat represents a complex matrix that may contain a variety of contaminants, some of which being involved in colon cancer development according to a commonly accepted hypothesis involving the formation of DNA adducts. 7,8

In terms of food safety, the cooking of meat at high temperature induces the formation of numerous electrophilic compounds from heterocyclic amines (HCAs) and polycyclic aromatic hydrocarbons (PAHs), such as 2-amino-3,8-dimethylimidazo-[4,5-f]-quinoxaline (MelQx) or benzo[a]pyrene (BaP), respectively, which are known to be pro-mutagenic by giving rise to DNA adducts after the formation of reactive metabolites. ${ }^{9}$ Alternatively, curing processes lead to the formation of $\mathrm{N}$-nitroso compounds (NOCs). ${ }^{10}$ Some of these NOCs are known to be mutagenic because of their ability to form DNA adducts. ${ }^{11}$ Finally, several studies showed convincing evidence that heme iron contained in red meat, could be one of the main factors of carcinogenesis promotion. ${ }^{12-14}$ The main hypothesis to explain 
heme iron toxicity relies on its ability to promote the formation of radical species by Fenton-like reactions, which leads to the peroxidation of dietary polyunsaturated fatty acids into secondary lipid peroxidation electrophilic products such as 4-hydroxynonenal (4-HNE). ${ }^{15}$ As reported by Sesink et al. ${ }^{16}$ and further confirmed by Bastide et al., ${ }^{17}$ heme-iron as such does not participate to carcinogenesis promotion, which is mainly mediated through lipid peroxidation product formation in the colon lumen. Targeted methods are commonly used to monitor these reactive metabolites, able to covalently bind to biomolecules..$^{18}$ Concerning lipid peroxidation, 4-HNE has been one of the most studied lipid peroxidation secondary product. ${ }^{19-21}$ However, depending on the fatty acid precursor, lipid peroxidation may induce the formation of many other reactive compounds (potentially unknown) with various abundance and reactivity. Developing untargeted methods are then necessary to get a more comprehensive view of their formation.

One of the main detoxification routes employed to detoxify such reactive/electrophilic compounds is the enzymatic conjugation with glutathione, a tripeptide known as an efficient scavenger of electrophilic species. Those glutathione conjugates are then cleaved by $\gamma$-glutamyltransferase and cysteinylglycine dipeptidase, respectively, and finally $N$-acetylated to form mercapturic acid conjugates (MACs) that are mostly urine-excreted. ${ }^{22}$ Thus, MACs are the result of detoxification processes of reactive electrophilic species and can be considered as biomarkers of exposure to these harmful compounds. Monitoring MACs could therefore be useful for a more accurate characterization of exposure to reactive compounds and a better evaluation of the role of reactive metabolites in toxicology in general..$^{23}$

Despite the interest of this approach, only a few studies have focused on the global analysis of MACs. ${ }^{24-}$ ${ }^{26}$ These studies aimed at evaluating the electrophilic burden of the organism by screening mercapturates in an untargeted way by liquid chromatography coupled with mass spectrometry (LCMS) using constant neutral loss-like acquisition mode. Other available studies of MACs were based on MS targeted analysis to study specific exposure situations like atrazine ${ }^{27}$ or neo-formed products in food such as acrylamide or acrolein. ${ }^{28}$ All these works use the characteristic loss of 129 Da observed 
87 from MACs in urine samples by the cleavage of the thioether bond, and rely on low-resolution mass 88 spectrometry.

89 In the present study, we present an original method for the global screening of MACs. A high-resolution 90 mass spectrometry (HRMS) based workflow was developed using a data-independent acquisition 91 mode (DIA) for monitoring the specific neutral-loss of mercapturates through gas phase fragmentation 92 in mass spectrometry. As a proof of concept, this paper presents results from a study based on an 93 animal experiment conducted with different diets containing various oils (fish oil, safflower oil and 94 hydrogenated coconut oil) with different polyunsaturated fatty acids profiles, in combination with 95 heme iron to catalyze lipid peroxidation. Thus, monitoring urinary MACs in these animals provided an 96 accurate overview of their exposure to lipid peroxidation products conjugated with glutathione.

\section{EXPERIMENTAL SECTION}

\section{Chemicals}

99 All solvents were purchased from Fisher Scientific (Thermo Fisher Scientific, Illkirch, France) and were 100 LC-MS grade. Standard 4-hydroxynonan-1-ol mercapturic acid (DHN-MA), 4-hydroxyoctan-1-ol-MA, 4101 hydroxyheptan-1-ol-MA， 4-hydroxyhexan-1-ol-MA (DHH-MA), 4-hydroxynonanal-MA, 4102 hydroxyoctanal-MA and 4-hydroxyheptanal-MA, were synthetized in the laboratory as previously 103 described $^{29}$. Standard $N$-acetyl-S-phenylcysteine was purchased from Sigma-Aldrich (Saint Quentin 104 Fallavier, France) and the MS calibration mix solution was purchased from Waters (Manchester, UK). 105 Heme iron (Hemin from bovine) was purchased from Sigma-Aldrich, and fish oil (Refined Menhaden 106 oil), safflower oil and hydrogenated coconut oil came from MP Biomedicals (Illkirch-Graffenstaden, 107 France).

\section{Animals and diets}

109 Young adults Fischer male rats (6 rats/group) were housed individually in plastic metabolic cages. They 110 were allowed for 3 days of acclimatization to their cage. The room was kept at a temperature of $22{ }^{\circ} \mathrm{C}$ 111 on a $12 \mathrm{~h}$ light-dark cycles. Animals had free access to tap water and to their respective diet for 15 days. 112 Diets were given each day at the end of the afternoon in order to limit oxidation, and 24h-urine 
113

114

115

116

117

118

119

120

121

122

123

124

125

126

127

128

129

130

131

132

133

134

135

136

137

138

samples were collected at day 12 and frozen $\left(-20^{\circ} \mathrm{C}\right)$ until analysis. A first set of diets contained $5 \%$ fish oil (containing long chain $\omega-3$ fatty acids). A second set of diets contained $5 \%$ safflower oil (containing $\omega-6$ fatty acids). A last set of control diets contained $5 \%$ hydrogenated coconut oil (containing almost only saturated fatty acids). All diets contained heme iron $(0.094 \% \mathrm{w} / \mathrm{w})$ to catalyze lipid peroxidation. All animal experiments were performed in accordance with EU directive 2010/63/EU and approved by the local Animal Care and Use Committee of Toulouse Midi-Pyrénées (agreement TOXCOM/0006/FG).

\section{Sample preparation}

Urine samples were allowed to thaw at room temperature. Aliquots of $500 \mu \mathrm{L}$ were prepared for each urine sample and diluted in $500 \mu \mathrm{L}$ of mobile phase $\mathrm{A}\left(95 \% \mathrm{H}_{2} \mathrm{O}, 5 \% \mathrm{CH}_{3} \mathrm{OH}, 0.1 \% \mathrm{CH}_{3} \mathrm{CO}_{2} \mathrm{H}\right)$. After dilution, the aliquots were vortex-mixed and centrifuged at 10,000 rpm for 5 minutes. The supernatants were transferred into vials to be directly used for analyses. This sample preparation protocol was previously developed for mass spectrometric detection of urinary metabolites of xenobiotics, including conjugated metabolites. ${ }^{30}$

\section{Liquid chromatography and mass spectrometry analyses}

Analyses were performed using ultra performance liquid chromatography (UPLC) (ACQUITY, Waters Manchester, UK) coupled to a quadrupole time-of-flight mass spectrometer (Q-ToF Synapt G2-Si, Waters, Manchester, UK). A volume of $10 \mu \mathrm{L}$ of samples were injected into a Hypersil Gold C18 (1.9 $\mu \mathrm{m}, 100 \times 2.1 \mathrm{~mm}$ ) analytical column (Thermo Fisher Scientific, Illkirch, France) at a flow rate of 0.3 $\mathrm{mL} / \mathrm{min}$, and maintained at $40^{\circ} \mathrm{C}$. According to a previously developed chromatographic protocol ${ }^{30}, \mathrm{a}$ linear gradient program was set up with mobile phase A: $95 \% \mathrm{H} 2 \mathrm{O} / 5 \%$ methanol / $0.1 \%$ acetic acid, and mobile phase B: $100 \%$ methanol / $0.1 \%$ acetic acid. Initial conditions were $100 \%$ of A, followed by a linear gradient from 0 to $100 \%$ of $B$ in $30 \mathrm{~min}$. These conditions were held for $4 \mathrm{~min}$ prior to switching in $1 \mathrm{~min}$ to the starting conditions and held for $5 \mathrm{~min}$ to equilibrate the column. MS ${ }^{\mathrm{E}}$ acquisitions (i.e. DIA mode) were achieved with electrospray ionization (ESI) in the negative mode. Parameters were set-up using two standard compounds (namely DHN-MA and N-acetyl-S-phenylcysteine) as detailed in the experimental section: capillary voltage $0.5 \mathrm{kV}$, sampling cone voltage $30 \mathrm{~V}$, source temperature 
$139120^{\circ} \mathrm{C}$, desolvation temperature $550{ }^{\circ} \mathrm{C}$, cone gas flow rate $30 \mathrm{~L} / \mathrm{h}$, desolvation gas flow rate $600 \mathrm{~L} / \mathrm{h}$ 140 and a trap collision energy of $15 \mathrm{eV}$ for the high energy spectra. MSE spectra were acquired with a 141 range of mass-to-charge ratio $(\mathrm{m} / \mathrm{z})$ set to $50-800 . \mathrm{MS}^{3}$ experiments were achieved on a LTQ Orbitrap 142 XL mass spectrometer (Thermo Scientific, Les Ulis, France).

143 A quality control (QC) sample corresponding to the pool of all samples was analyzed repetitively $(n=$ 144 7) along the injection sequence to ensure that no instrumental deviation was observed during the 145 analysis. The quality of analyses was checked by monitoring the variation the chromatographic 146 pressure (lower than 10 bars between the first and the last injection), the variation of MS signal 147 (Relative Standard Deviation (RSD) of signal area of $8.6 \%$ ), retention time (RSD of $0.15 \%$ ) and mass 148 measurement accuracy (lower than $5 \mathrm{ppm}$ ) based on the tryptophan signal detected at 3.2 min.

\section{Data processing}

150 Data from MS ${ }^{\mathrm{E}}$ experiments were processed with the UNIFI ${ }^{\circledR}$ software (Waters, Manchester, UK). 151 Extraction of data from full scan mass spectra was carried out using XCMS offline software ${ }^{31}$ with 152 centwave algorithm ${ }^{32}(\mathrm{ppm}=10$, peakwidth $=(10,70)$, snthresh $=10$, noise $=2000, \mathrm{bw}=5, \mathrm{mzwid}=$ 153 0.01). To avoid urine dilution variation among samples data were normalized using Probabilistic 154 Quotient Normalization. ${ }^{33}$ All data analysis steps were performed using workflow4metabolomics, a 155 collaborative portal dedicated to metabolomics data processing. ${ }^{34}$

156 Statistical analyses were carried out using SIMCA software v15 (Umetrics, Umeå, Sweden). Results 157 were examined using unsupervised principal component analysis (PCA) in order to check the validity 158 of acquisition and to detect potential outliers. Projection to latent structure discriminant analysis 159 modeling (PLS-DA) was then carried out to find out diet discriminant features. The PLS-DA $\mathrm{Q}^{2}$ 160 parameter (fraction of samples correctly predicted by the model) was used to assess the quality of 161 models. Models with $\mathrm{Q}^{2}>0.4$ were considered as valid. For valid models, a permutation test was 162 performed to assess the robustness of models. For valid and robust models, significant features were 163 selected by their variable importance on projection (VIP). A feature displaying a VIP $>1$ was considered 164 significant. 
RESULTS AND DISCUSSION

166

167

168

169

170

171

172

173

174

175

176

177

178

179

180

181

182

183

184

185

186

187

188

189

190

\section{Method development}

To setup ionization parameters for the profiling of MACs, two standards were used. The first one corresponds to the reduced conjugate between $N$-acetylcysteine and 4-HNE, namely DHN-MA. Since this compound is generated from lipid peroxidation, it is representative of structures we would like to detect. The second standard corresponds to a smaller structure, which displays no heteroatom other than those of the $N$-acetylcysteine moiety, namely $N$-acetyl-S-phenylcysteine, the conjugate between a phenyl group and $\mathrm{N}$-acetylcysteine. This compound could be considered as representative of smaller and less ionizable compounds.

UPLC coupled with HRMS through ESI is an analytical approach particularly suited for the qualitative and quantitative analysis of urinary metabolites. ${ }^{5} \mathrm{ESI}$ in the negative mode gave the most intense signal, due to the carboxylic acid function of the mercapturate that easily undergoes deprotonation. Fragment ions were observed in the full MS spectrum of standards even with no collision energy applied into the collision cell of the mass spectrometer. The [M-H]- ion of DHN-MA was detected at $m / z$ 320.1531, but another intense ion was observed at $m / z 191.1111$, corresponding to the loss of $129 \mathrm{Da}$, representative of the $\mathrm{N}$-acetylcysteine moiety. The same phenomenon was observed with $\mathrm{N}$ acetyl-S-phenylcysteine. This fragmentation was caused by an excess of internal energy of ions coming from the desolvation conditions of the electrospray ion source and the conditions of transfer of the ions into the Triwave and StepWave cells of the Synapt mass spectrometer. Nevertheless, this fragmentation has to be limited to increase the signal of the deprotonated ions of MACs, and therefore to increase the sensitivity of the method. For that purpose, we decreased the excess of internal energy during the steps of ionization / desolvation and ion transfer by limiting the acceleration of ions into the different cells of the Synapt mass spectrometer where remaining gas was present, i.e. into the StepWave cell for the last step of desolvation and into the Triwave cell for the transfer of ions.

We based our profiling method on the property of MACs to undergo a particular fragmentation during the collision induced dissociation (CID) process, leading to a neutral loss of $\mathrm{C}_{5} \mathrm{H}_{7} \mathrm{NO}_{3} 129.0426 \mathrm{Da}$, 
191 corresponding to the cleavage of the thioether bond as already mentioned in previous works. ${ }^{24-28}$ In 192 this study, this fragmentation pattern was investigated from seven MAC standard compounds 193 originating from lipid peroxidation products conjugated with glutathione, namely 4-hydroxynonan-1194 ol mercapturic acid (DHN-MA), 4-hydroxyoctan-1-ol-MA, 4-hydroxyheptan-1-ol-MA, 4-hydroxyhexan1-ol-MA (DHH-MA), 4-hydroxynonanal-MA, 4-hydroxyoctanal-MA and 4-hydroxyheptanal-MA, as well as the standard of $\mathrm{N}$-acetyl-S-phenylcysteine. Results summarized in Table 1 show that all MACs displayed the neutral loss of $\mathrm{C}_{5} \mathrm{H}_{7} \mathrm{NO}_{3}$ 129.0426 Da. As an example, in the case of DHN-MA detected at a retention time $(\mathrm{Rt})$ of $13.2 \mathrm{~min}$, the neutral loss of $129.0426 \mathrm{Da}$ was observed with a resulting fragment ion at $\mathrm{m} / \mathrm{z}$ 191.1106. Consecutive losses of $\mathrm{H}_{2} \mathrm{O}$ and $\mathrm{CH}_{2} \mathrm{O}$ led to the formation of $\mathrm{m} / \mathrm{z}$ 173.1000 and 143.0898 ions. The complementary fragment ion $\left[\mathrm{C}_{5} \mathrm{H}_{7} \mathrm{NO}_{3}-\mathrm{H}\right]^{-}$detected at $\mathrm{m} / \mathrm{z}$ 128.0352, was also previously observed for three metabolites of $\mathrm{B}(\mathrm{a}) \mathrm{P}^{35}$ and used as multiple reaction monitoring transition (MRM) for detecting some MACs. ${ }^{36}$ However, this ion was observed only in the MS/MS spectra of DHH-MA and DHN-MA, and with a weak relative abundance $(<3 \%)$.

Table 1: UHPLC-ESI-HRMS/MS of MAC standards, achieved at a collision energy of $15 \mathrm{eV}$.

\begin{tabular}{|c|c|c|c|c|}
\hline MAC & $m / z$ & $\begin{array}{l}\text { Rt } \\
\text { (min) }\end{array}$ & $\begin{array}{l}\text { Neutral } \\
\text { Formula }\end{array}$ & Fragment Ions (Relative Abondance) \\
\hline 4-hydroxyheptanal-MA & 290.1067 & 7.74 & $\mathrm{C} 12 \mathrm{H} 21 \mathrm{NO} 5 \mathrm{~S}$ & $\begin{array}{l}290.1061(20) 161.0637 \text { (53) } 143.0532 \\
(100) 125.0426(3) 115.0579(25)\end{array}$ \\
\hline 4-hydroxyoctanal-MA & 304.1223 & 10.67 & $\mathrm{C} 13 \mathrm{H} 23 \mathrm{NO} 5 \mathrm{~S}$ & 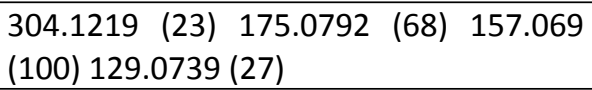 \\
\hline $\begin{array}{l}\text { 4-hydroxynonanal-MA } \\
\text { (HNE-MA) }\end{array}$ & 318.138 & 13.18 & $\mathrm{C} 14 \mathrm{H} 25 \mathrm{NO} 5 \mathrm{~S}$ & $\begin{array}{l}318.1383(16) 189.0946(25) 171.0839 \\
(100) 143.0880(17)\end{array}$ \\
\hline 4-hydroxyheptanol-MA & 292.1223 & 7.35 & $\mathrm{C} 12 \mathrm{H} 23 \mathrm{NO} 5 \mathrm{~S}$ & $\begin{array}{l}292.1219(30) 163.079(100) 145.049(34) \\
130.963(10) 115.0583(39)\end{array}$ \\
\hline 4-hydroxyoctanol-MA & 306.138 & 10.27 & $\mathrm{C} 13 \mathrm{H} 25 \mathrm{NO} 5 \mathrm{~S}$ & $\begin{array}{l}306.1373(27) 177.0949(100) 159.0847 \\
(23) 129.0741(32)\end{array}$ \\
\hline $\begin{array}{l}\text { 4-hydroxyhexanol-MA } \\
\text { (DHH-MA) }\end{array}$ & 278.1068 & 4.47 & $\mathrm{C} 11 \mathrm{H} 21 \mathrm{NO} 5 \mathrm{~S}$ & $\begin{array}{l}278.1062(20) 149.0637(100) 131.053 \\
(38) 128.0346(3) 101.0424(42)\end{array}$ \\
\hline $\begin{array}{l}\text { 4-hydroxynonanol-MA } \\
\text { (DHN-MA) }\end{array}$ & 320.15374 & 13.21 & C14H27NO5S & $\begin{array}{l}320.1536(4) 191.1106 \text { (100) } 173.1000 \\
(16) 143.0898(44) 128.0352(2)\end{array}$ \\
\hline$N$-acetylphenylcysteine & 238.0543 & 9.92 & C11H13N03S & $109,0112(100)$ \\
\hline
\end{tabular}

We applied a data-independent acquisition (DIA) process allowing the fragmentation of all ions. In the 
209 first scan is used to detect the precursor ion, whereas the second scan is used to detect the fragment 210 ions. ${ }^{37}$ Data are then processed using the UNIFI ${ }^{\circledR}$ software to associate the two scans, and to build 211 product ion spectra from the collected data. Within these data, the software also offers the possibility 212 to screen every ions giving a particular neutral loss. The neutral loss tolerance was set at $5 \mathrm{mDa}$ as it 213 was the value which gave the most notable results without being too large. The signal threshold of the 214 low energy scan was set at 2,000 counts to minimize the signals mixed to the background noise 215 whereas the signal threshold of the high energy scan was set at 20 counts. At the end, only candidates 216 displaying a neutral loss or a specific fragment ion detected with a mass accuracy below 10 ppm were 217 kept for subsequent processing.

218 The limit of detection (LOD) of the method was evaluated by analyzing successive dilutions of DHN$219 \mathrm{MA}$ and $\mathrm{N}$-acetyl-S-phenylcysteine standard solutions prepared in blank urine samples. A signal ratio 220 of 3 was observed at concentrations of $5 \mathrm{ng} / \mathrm{mL}$ and $10 \mathrm{ng} / \mathrm{mL}$ for DHN-MA and N-acetyl-S221 phenylcysteine, respectively. Although the dynamic range of the detector is limited when working in 222 the profile mode, it can be increased by using the dynamic range extension (DRE) in the centroid mode. 223 Since the centroid mode is not compatible with $\mathrm{UNIFI}^{\circledR}$, a parallel untargeted acquisition of data in the 224 centroid mode should be performed for quantitative purposes. Thus, according to the workflow 225 displayed in Figure 2, all samples were analyzed by UPLC-ESI-HRMS, and raw data were processed using 226 XCMS. ${ }^{31}$ In parallel and using exactly the same chromatographic and ionization parameters, QC 227 samples were also analyzed by UPLC-ESI-HRMSE to detect MACs. Features of MACs were then 228 annotated into the XCMS dataset to allow their statistical analyses using unsupervised or supervised 229 statistical tools.

230 In DIA mode, no precursor ion is selected and fragmentation patterns are built in-silico. Thus, false 231 positive MACs might be detected by the profiling of the considered neutral loss. A first possible origin 232 of artefactual detection could be chromatography. However, since features detected by DIA are 233 matched to features detected by XCMS, only features displaying chromatographic characteristics in 234 agreement with parameters used for XCMS (i.e. signal to noise ratio, peak width, peak resolution) are 
235 kept. The second origin of potential false positive MACs could be the non-specificity of the 236 fragmentation (i.e., neutral loss of $\mathrm{C}_{5} \mathrm{H}_{7} \mathrm{NO}_{3}$ 129.0426 Da not specific of MACs), or if the deconvolution 237 algorithm of DIA is not enough robust to align the right fragment ion with the right precursor ion. 238 However, since next steps of the method aim at highlighting discriminant features with statistical tools, 239 and then identifying them with targeted MS/MS, false positive MACs will be eliminated at this last 240 step.

241

242

\section{step.}

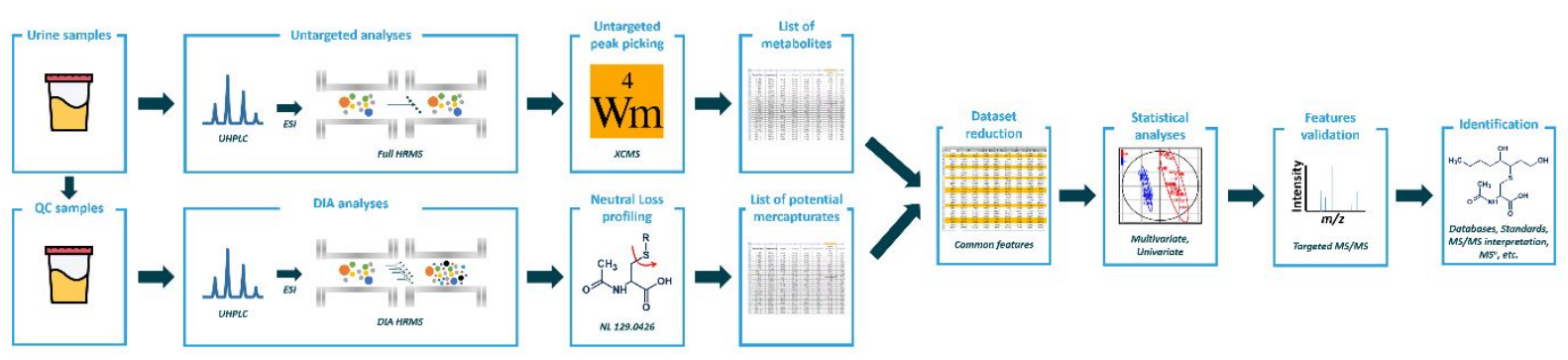

Figure 1. Workflow of the untargeted profiling of mercapturic acid conjugates.

\section{Application}

This method was validated on a proof-of-concept application on urine samples coming from rats fed diets containing representative lipid peroxidation prone fatty acids expected to yield various glutathione conjugates, further metabolized into MACs. Thus, the global profiling method of MACs should be more adapted to detect MACs by comparison with an untargeted metabolomics approach. Since lipid peroxidation products depend on their precursor unsaturated fatty acid, three different diets were selected. The first one was based on hydrogenated coconut oil known to contain almost only saturated fatty acids and which should produce no MACs (except those possibly observed coming from endogenous peroxidation), the second one was based on fish oil containing long chain omega-3 fatty acids, and finally the third one was based on safflower oil, which is rich in omega-6 fatty acids. Thus these three contrasted diets were expected to lead to specific peroxidation products, that are detoxified by conjugation with glutathione, and finally eliminated as specific MACs in urine. Urine samples coming from the different diets were randomly analyzed by UPLC-ESI-HRMS. Since the detection of standard MACs analyzed for the set-up of the method was more sensitive in the negative ionization mode, samples were analyzed only with this mode. After data acquisition, features were 
258

259

260

261

262

263

264

265

266

267

268

269

270

271

272

273

274

detected using XCMS. Raw data were filtered according to a blank sample of mobile phase A, to keep only features whose signal intensity is at least three times higher in the QC samples than in the blank samples. A total of 9614 features were detected by this untargeted metabolomics approach (Supporting information, Table S1). In parallel, the QC sample was also analyzed according to our designed $\mathrm{MS}^{\mathrm{E}}$ workflow. By MS' eighty-two MACs candidates were detected. Twenty-six of them displayed poor chromatographic characteristics, including a low signal to noise ratio, a large peak width or a very low chromatographic resolution. All these false positive MACs arising from chromatography were not present in the XCMS data matrix (Supporting information, Table S2) illustrating the relevant data curation achieved by using a parallel processing of data using XCMS.

An unsupervised statistical analysis by PCA was achieved on all the features detected with XCMS in all samples and in the QC. No outlier sample was detected, and a good correlation of the injections of the QC sample demonstrated that no deviation has occurred along the analysis (Supporting information, FigureS1). The resulting PCA shows in Figure 2A a discrimination of the fish oil diet (FO), but a weak separation of coconut oil diet (CO) and safflower oil diet (SA). By comparison, the same PCA achieved on features filtered thanks to our MSE approach obviously displays a better discrimination of diets (Figure 2B). Results match perfectly the expected effects of the lipid peroxidation induced by heme iron according to the composition of oil in poly-unsaturated fatty acids. 


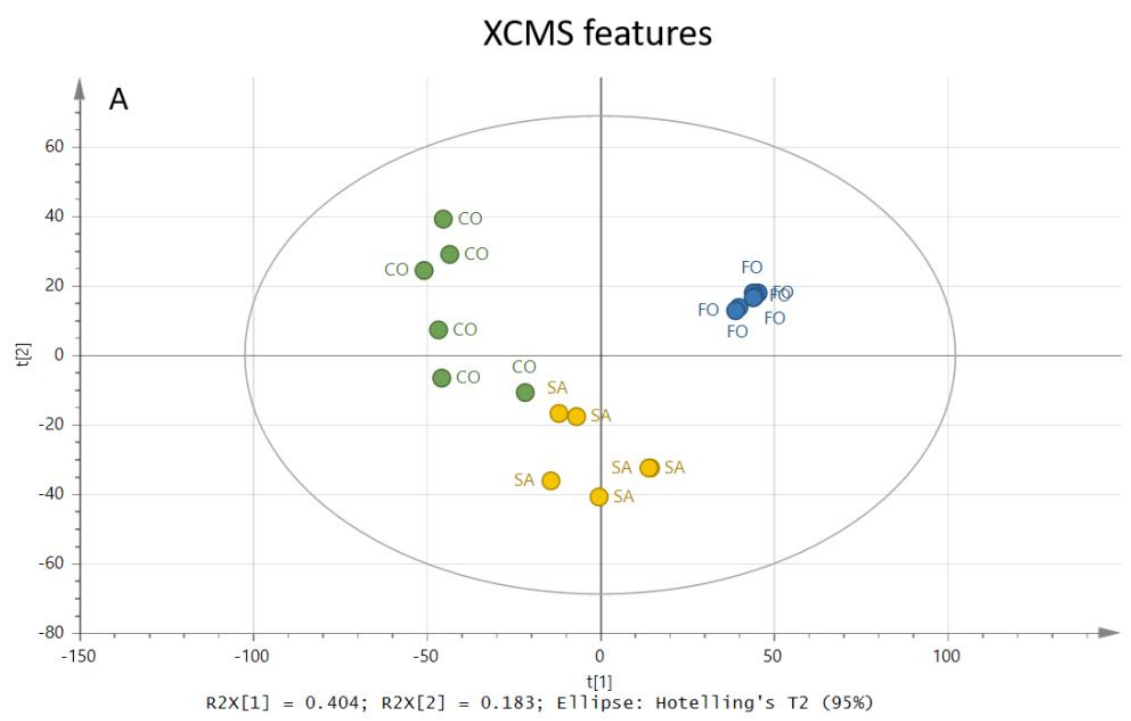

MSE features

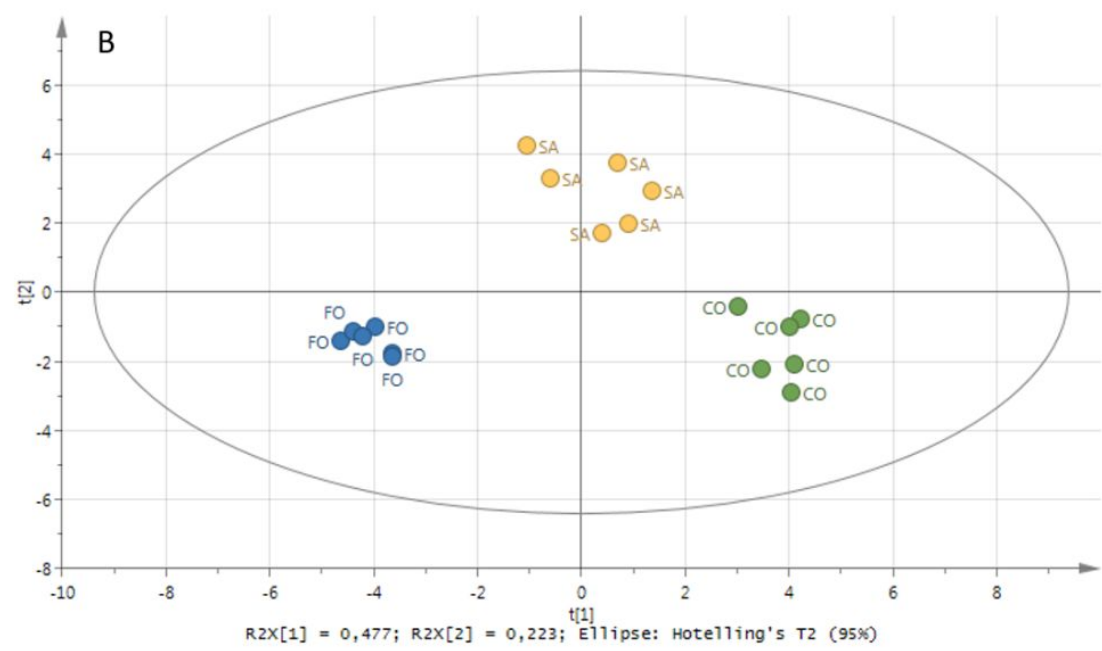

275

276 Figure 2. Principal Component Analyses of (A) all features detected with XCMS and (B) features 277 detected by MSE. Yellow dots (SA) represent samples from safflower oil diet, blue dots (FO) represent 278 samples from fish oil diet, and green dots (CO) represent coconut oil diet.

279 To evaluate the contribution of the $\mathrm{MS}^{\mathrm{E}}$ profiling approach to discriminate populations, PLS-DA were 280 achieved on both dataset validated by permutation tests. Samples were discriminated according to a 281 two components model $\left(Q^{2}=0.94\right)$ of 3944 features with a VIP $>1$ from the dataset generated by XCMS (Supporting information, FigureS2). Conversely, a two components model $\left(Q^{2}=0.95\right)$ of 22 features with a VIP > 1 was obtained from the dataset generated by $\mathrm{MS}^{\mathrm{E}}$ (Supporting information, FigureS3). In the XCMS dataset, discriminating MACs were ranked between the 21 and 4354 position 
286 Table S3). This shows that the discriminating MACs were diluted into thousands of features 287 representative of other metabolites using the whole metabolomics dataset, making it very difficult to 288 identify them. At the opposite, the PLS-DA achieved on the MSE dataset allowed to directly highlight 289 discriminating MACs and to detect two more significant MACs. This better discrimination can be explained by the fact that the drastic data matrix reduction, enabling to focus the dataset only on a class of metabolites of interest. The studied populations were expected to be discriminated according to the lipid peroxidation products, detoxified by conjugation with glutathione and finally metabolized into MACs. Therefore, by looking only at MACs, which include lipid peroxidation products conjugates, the data matrix was filtered only on metabolites of interest, excluding other potential interfering signals.

Another interesting issue of the analysis of metabolites, is the identification of discriminating compounds. Since a fragmentation pattern highly specific of MACs is monitored, this identification criteria can be proposed in a first attempt. To validate the loss of 129.0425 as representative of MACs, targeted MS/MS analyses were attempted on the 56 MACs candidates detected in the XCMS data matrix. Some MS/MS spectra could not be generated likely due to a loss of sensitivity related to the decrease of the scan time which has to be set to allow numerous parallel MS/MS acquisitions, and/or the weak signal of corresponding precursor ions. In all others MS/MS spectra, the expected neutral loss was successfully observed, showing that no interference occurred during its detection by MSE. To assess the possible detection of a potential loss of 129.0425 from a metabolite that was not a MAC, the chemical formula of each candidate was generated. Only two candidates displayed a loss of 129.0425 from a chemical formula bearing no sulfur atom (n230.1032T4.9 and n261.0879T5.7). The list of features detected by MSE was also matched with the HMDB database. Some features matched with metabolites that are not a MAC. However, none of these other potential metabolites had MS/MS spectra in HMDB displaying a [(M-H) - 129]- fragment ion.

310 Based on this proposed identification of MACs, a further hypothesis of the reactive metabolite before 311 its conjugation with glutathione can also be formulated (Table 2), with a particular focus on lipid 
312 peroxidation products in this study. A chemical formula of the precursor reactive metabolite can be

313 hypothesized from the formula of the corresponding MAC, to which the $N$-acetylcysteine formula was

314 subtracted. Then, putative lipid peroxidation products were matched with the LIPID MAPS database.

315 In the case of unknowns or not well characterized lipid peroxidation products, annotation was

316 performed from bibliography of lipid peroxidation products. ${ }^{29,38,39}$

317 Table 2. Annotated MACS of lipid peroxidation products detected by UPLC-ESI-HRMSE in urine

318 samples of rats

\begin{tabular}{|c|c|c|c|c|c|c|c|}
\hline XCMS feature & $m / z$ & Rt (min) & {$[\mathrm{M}-\mathrm{H}]$ - Formula } & ppm & annotation of MACs & $\begin{array}{c}\text { Annotation of reactive } \\
\text { metabolite }\end{array}$ & $\begin{array}{c}\text { Identification } \\
\text { level }^{40}\end{array}$ \\
\hline n248.0955T6.5 & 248.0955 & 6.40 & C10H18NO4S & 2.8 & pentanol-MA & pentenol & 3 \\
\hline n262.1113T9.5 & 262.1114 & 9.51 & $\mathrm{C} 11 \mathrm{H} 2 \mathrm{ONO} 4 \mathrm{~S}$ & 1.6 & hexanol-MA & hexenol & 3 \\
\hline n266.0849T13.8 & 266.0847 & 13.78 & C13H16NO3S & 3.4 & ethylbenzene-MA & styrene & 3 \\
\hline $\mathrm{n} 274.0746 \mathrm{T6}$ & 274.0737 & 5.44 & C11H16NO5S & 6.6 & ethyllactone-MA & hydroxyhexenoic ac & 3 \\
\hline n276.0905T10 & 276.0908 & 10.02 & C11H18NO5S & 1.0 & 4-hydroxyhexanal-MA (HHE-MA) & 4-hydroxyhexenal (HHE) & 2 \\
\hline n276.1271T12.5 & 276.1270 & 12.50 & $\mathrm{C} 12 \mathrm{H} 22 \mathrm{NO} 4 \mathrm{~S}$ & 1.8 & heptanol-MA & heptenol & 3 \\
\hline \begin{tabular}{|l|} 
n278.1054T4.6 \\
\end{tabular} & 278.1059 & 4.52 & C11H2ONO5S & 3.1 & 4-hydroxyhexanol-MA (DHH-MA) & 4-hydroxyhexenol (DHH) & 1 \\
\hline \begin{tabular}{|l|} 
n278.1054T5.6 \\
\end{tabular} & 278.1062 & 4.64 & C11H2ONO5S & 2.0 & hydroxyhexanol-MA & hydroxyhexenol & 3 \\
\hline n288.1269T13.2 & 288.1271 & 13.17 & $\mathrm{C} 13 \mathrm{H} 22 \mathrm{NO} 4 \mathrm{~S}$ & 1.5 & octanal-MA & octenal & 3 \\
\hline \begin{tabular}{|l|} 
n288.1269T12.5 \\
\end{tabular} & 288.1269 & 12.81 & $\mathrm{C} 13 \mathrm{H} 22 \mathrm{NO} 4 \mathrm{~S}$ & 2.1 & octanal-MA & octenal & 3 \\
\hline n288.1271T11.5 & 288.1268 & 11.50 & $\mathrm{C} 13 \mathrm{H} 22 \mathrm{NO} 4 \mathrm{~S}$ & 2.3 & octanal-MA & octenal & 3 \\
\hline n290.106T6.2 & 290.1057 & 6.07 & $\mathrm{C} 12 \mathrm{H} 20 \mathrm{NO} 5 \mathrm{~S}$ & 3.9 & hydroxyheptanal-MA & hydroxyheptenal & 3 \\
\hline n290.1425T15.2 & 290.1425 & 15.22 & $\mathrm{C} 13 \mathrm{H} 24 \mathrm{NO} 4 \mathrm{~S}$ & 2.0 & octanol-MA & octenol & 3 \\
\hline \begin{tabular}{|l|} 
n304.0855T4.2 \\
\end{tabular} & 304.0854 & 4.54 & C12H18NO6S & 2.0 & hydroxypropyllactone-MA & 4,7-dihydroxyheptenoic ac & 3 \\
\hline \begin{tabular}{|l|} 
n306.1019T4.7 \\
\end{tabular} & 306.1014 & 5.12 & C12H2ONO6S & 1.7 & hydroxyheptanoic ac-MA & hydroxyheptenoic ac & 3 \\
\hline \begin{tabular}{|l|} 
n314.1433T14.4 \\
\end{tabular} & 314.1424 & 14.45 & C15H24NO4S & 2.1 & decenal-MA & decadienal & 3 \\
\hline n318.1374T10.6 & 318.1375 & 10.70 & C14H24NO5S & 1.9 & hydroxynonenol-MA & hydroxynondienol & 3 \\
\hline n320.117T7.1 & 320.1170 & 7.20 & C13H22NO6S & 1.0 & dihydroxyoctanal-MA & dihydroxyoctenal & 3 \\
\hline \begin{tabular}{|l|} 
n320.1529T12.9 \\
\end{tabular} & 320.1530 & 13.07 & $\mathrm{C} 14 \mathrm{H} 26 \mathrm{NO} 5 \mathrm{~S}$ & 2.3 & 4-hydroxynonanol-MA (DHN-MA) & 4-hydroxynonenol (DHN) & 1 \\
\hline n336.1115T3.6 & 336.1114 & 3.41 & $\mathrm{C} 13 \mathrm{H} 22 \mathrm{NO} 7 \mathrm{~S}$ & 2.3 & dihydroxyoctanoic ac-MA & dihydroxyoctenoic ac & 3 \\
\hline \begin{tabular}{|l|} 
n362.1272T6.4 \\
\end{tabular} & 362.1274 & 6.34 & C15H24NO7S & 1.5 & wCOOH-hydroxydecanal-MA & wCOOH-hydroxydecenal & 3 \\
\hline
\end{tabular}

320 An interesting feature was detected at $\mathrm{m} / \mathrm{z} 320.1529$ and $\mathrm{Rt}=13.07 \mathrm{~min}$. This metabolite displayed a

$321 \mathrm{~m} / \mathrm{z}$ ratio consistent with a $\left[\mathrm{C}_{14} \mathrm{H}_{27} \mathrm{NO}_{5} \mathrm{~S}-\mathrm{H}\right]^{-}$ion with a precision of $2.3 \mathrm{ppm}$. Four fragment ions were

322 detected in its MS/MS spectrum (Figure 3B) at m/z 191.1100, 173.0997, 143.0894 and 128.0346. 
323 Relative abundances of these ions were 100\%, 23\%, 52\% and $2 \%$, respectively, which were highly 324 similar with those of standard DHN-MA (Figure 3A, Table1). These two independent and orthogonal 325 data (i.e. retention time and MS/MS spectrum) comparable with a standard analyzed under identical 326 experimental conditions allowed us to identify this metabolite as DHN-MA at a level 1 of identification, 327 in full concordance with the nomenclature of identification proposed by the metabolomics standards 328 initiative (MSI). ${ }^{40}$ Interestingly, the consecutive fragmentation of the $[(\mathrm{M}-\mathrm{H})-129]^{-}$ion of DHN-MA 329 produced the $\mathrm{m} / \mathrm{z} 143.0894$ fragment ion by losses of $\mathrm{H}_{2} \mathrm{O}$ and $\mathrm{CH}_{2} \mathrm{O}$. This loss of $\mathrm{CH}_{2} \mathrm{O}$ could be 330 representative of the primary hydroxyl function of DHN-MA, which could give some information on 331 the structure of others MACs.
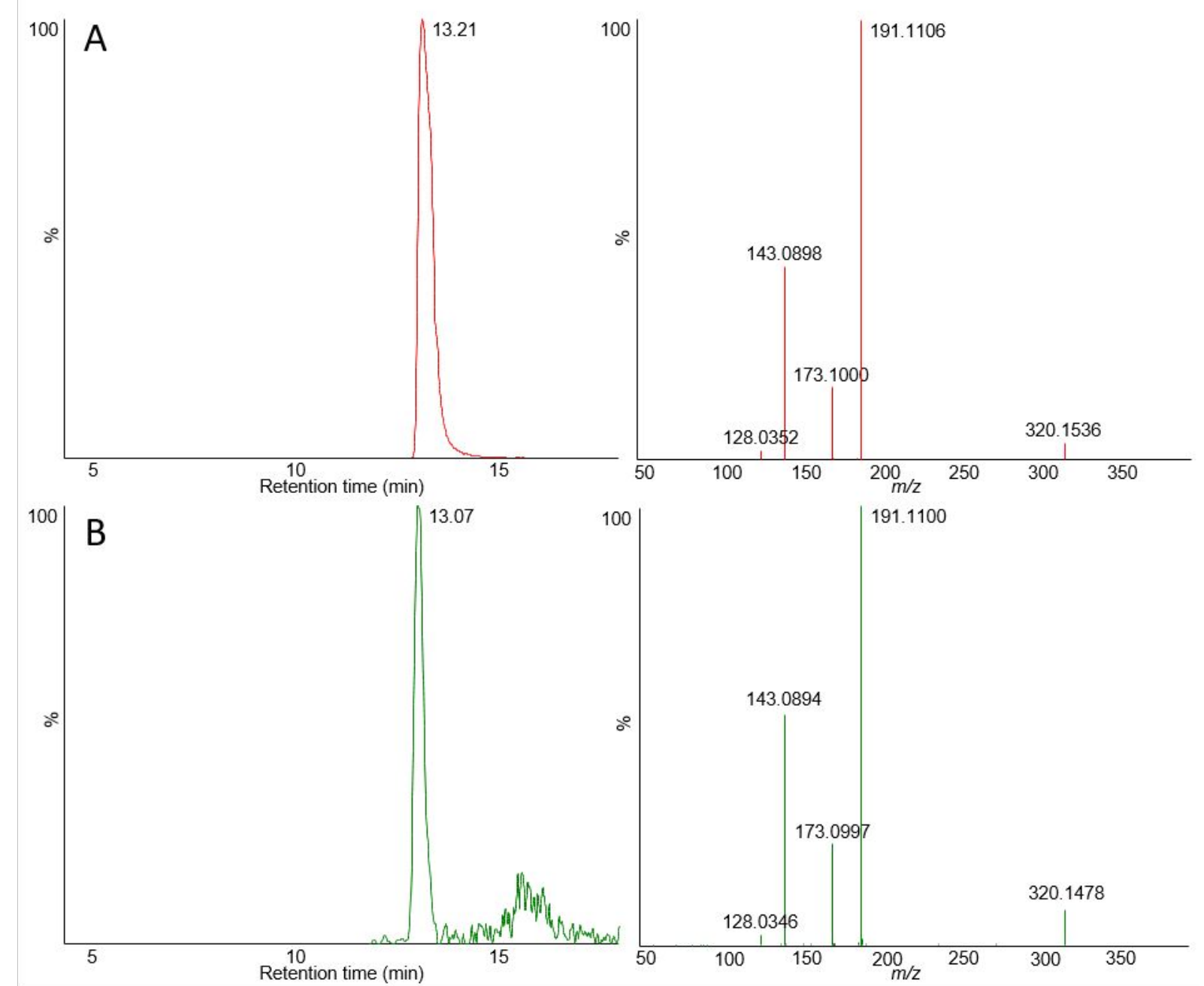

Figure 3: Extracted ion chromatogram and product ion spectrum at a collision energy of $15 \mathrm{eV}$, of $(A)$ the standard of DHN-MA, and (B) DHN-MA detected in urine samples. 
335 Related to DHN-MA, the mercapturate conjugate coming from the glutathione conjugation of HNE 336 (HNE-MA), before its metabolization into DHN-MA, could also be annotated (n318.1374T10.6 in Table 337 2). However, its targeted MS/MS spectrum (Supporting information, Table S2) and its retention time 338 did not match with synthetized standard of HNE-MA (Table 1). The consecutive fragmentation of the $339[(\mathrm{M}-\mathrm{H})-129]^{-}$ion of the standard produced the $m / z 143.0888$ fragment ion by losses of $\mathrm{H}_{2} \mathrm{O}$ and $\mathrm{CO}$. 340 This loss of CO could be representative of the aldehyde function of HNE-MA. By comparison, the 341 consecutive fragmentation of the $[(\mathrm{M}-\mathrm{H})-129]^{-}$ion of the unknown n318.1374T10.6 produced the $m / z 141.0749$ fragment ion by losses of $\mathrm{H}_{2} \mathrm{O}$ and $\mathrm{CH}_{2} \mathrm{O}$. This observation, which is similar with DHNMA, suggests that this unknown MAC may include a primary hydroxyl function, another hydroxyl function and an unsaturation, which can be a ring or a double bond.

Whereas DHN-MA is originating from $\omega-6$ polyunsaturated fatty acids, $\omega-3$ ones are known to lead to the formation of 4-hydroxyhexenal (HHE), which can be conjugated with glutathione to form HHE-MA.

${ }^{41}$ HHE-MA can be metabolized similarly to HNE-MA to generate the 4-hydroxyhexanal-MA (DHH-MA), which was detected at $\mathrm{m} / \mathrm{z} 278.1054$ and $\mathrm{Rt}=4.52 \mathrm{~min}$. This latter identification was confirmed at level 1 by analysis of the corresponding standard (Supporting information, FigureS4), which displayed the same retention time and fragmentation pattern. The mercapturate conjugate coming from the glutathione conjugation of HHE (HHE-MA), before its metabolization into DHH-MA, could also be annotated (n276.0905T10 in Table 2). Based on its MS/MS spectra (Supporting information, Table S2) and the biological context, the HHE-MA was putatively identified (level 2 , according to MSI ${ }^{40}$ ). The $m / z$ 290.1057 ion detected at Rt $=6.1 \mathrm{~min}$ (Supporting information, Table S2) was not attributed to 4hydroxyheptanal-MA, based on the MS/MS spectrum and Rt of the corresponding standard (Table 1). Some annotated MACs displayed a high degree of unsaturation, which can correspond to double bonds or rings. This latter possibility has already been described as lactone structures for lipid peroxidation 358 products. ${ }^{39}$ For example, the metabolite detected at $\mathrm{m} / \mathrm{z} 304.0854$ and $\mathrm{Rt}=4.5 \mathrm{~min}$ corresponds to a 359 conjugate formed between $N$-acetylcysteine and a structure displaying 7 carbon atoms, 3 oxygen 360 atoms, and 2 double bond equivalents. Since it is likely that this metabolite is a product of lipid 
361

362

363

364

365

366

367

368

369

370

371

372

373

374

375

376

377

378

379

380

381

382

383

384

385

386

peroxidation, one unsaturation may be involved in a carbonyl function at the carbon in position one.

Then the second unsaturation can be either a carbon-carbon double bond, or another carbonyl function. It is also possible that a dihydroxylated acid alken, hydroxylated in particular at position 4 can be conjugated as a dihydroxylated heptanoic acid mercapturate, which could further undergo a condensation to generate a lactone compound, like the hydroxypropylactone, according to previously described metabolic processes for HNE. ${ }^{38,39}$

As observed on the MS/MS spectra of standards (Table 1), MACs display poor fragmentation rates, which limits available information for unknowns identification. Neutral loss profiling was used to give a first step of identification (i.e. MAC), and then statistics on particular population highlighted some particular metabolites to have another hypothesis of identification (i.e. lipid peroxidation products). Based on these hypotheses and since standard MACs are not commercially available, seven chemical syntheses were achieved, among which five did not validate corresponding putative annotations. Some consecutive fragmentations could occur from the [(M - H -129$]^{-}$fragment ion, but the weak signal of the precursor ion prevented their detection in many cases (Supporting information, Table S3). The use of $\mathrm{MS}^{3}$ experiments could be an alternative solution to get more structural information. However, the analysis of the samples on a LTQ Orbitrap XL mass spectrometer led to the detection of only DHH-MA, hydroxynonenol-MA, DHN-MA, dihydroxyoctanal-MA and dihydroxyoctanoic ac-MA, and the corresponding $\mathrm{MS}^{3}$ spectra displayed only a loss of water.

\section{CONCLUSIONS}

Urinary MACs are originated from detoxification conjugates between glutathione and reactive electrophilic compounds that are able to bind to biomolecules, such as DNA and proteins and to induce deleterious effects. These compounds can be electrophilic as such or be the result of the bioactivation of xenobiotics to which we are exposed. Therefore, by monitoring MACs into urine, it is possible to monitor their precursor electrophilic metabolites and by extension, the molecules from which these metabolites are produced and which can be considered as toxicologically relevant compounds. The global profiling of MACs combining the detection of known and unknown MACs by using MSE, and their 
387 relative quantification, pave the way for addressing new perspectives in toxicology. The application of 388 this method to the study of lipid peroxidation catalyzed by heme iron, allowed highlighting various 389 unknown structures of reactive lipid peroxidation products, which may be implicated in the promotion 390 of colorectal cancer, similarly to $4-\mathrm{HNE}^{42}$. This detection of unknown potentially reactive metabolites 391 was greatly facilitated by the simplification of statistical analyses due to dataset reduction provided by the specific monitoring of metabolites of interest using the MSE profiling. Although our workflow suffers from some complexity because of the difficulty to obtained large amount of quantitative data with the software we used for DIA processing, this approach could be adapted to other mass spectrometers, DIA approaches, or fast data dependent acquisitions.

This case study was only focused on the study of MACs to demonstrate the proof of concept. However, the approach could be applied to monitor other classes of metabolites and markers, such as conjugates with glucuronic acid, sulfate, cysteine, glutathione, as well as DNA and RNA adducts as recently published, ${ }^{43}$ which are all displaying characteristic neutral losses.

400 Furthermore, it is noteworthy that this method is providing not only the profiling of MACs by MSE, but 401 also in parallel an untargeted analysis of the metabolome by HRMS, in the same samples. Indeed, an untargeted metabolomics analysis by reversed phase liquid chromatography coupled with HRMS using 403 electrospray ionization is able to detect amino acids, organic acids, polyols, nucleosides, indoles, etc. 44, which can give access to the metabolome modifications induced by the studied diets. Therefore, this method allows characterizing a series of toxicologically relevant compounds in one hand, as well as the study of their effects on the metabolome in the other hand, in the same analysis of samples. This may represent a valuable way to link exposure to toxics and possible health adverse outcomes.

\section{ACKNOWLEDGMENTS}

PhD doctoral fellowship of LM was co-funded by INRAE (Nutrition, Chemical Food Safety and Consumer

410 Behaviour Scientific Division) and French region Occitanie. All MS experiments were performed on the 411 instruments of the MetaToul-AXIOM platform, partner of the national infrastructure of metabolomics 412 and fluxomics: MetaboHUB [MetaboHUB-ANR-11-INBS-0010, 2011]. 


\section{ASSOCIATED CONTENT}

\section{Supporting Information}

415 Table S1: Data matrix obtained form UPLC-ESI-HRMS using XCMS; Table S2: Candidates of MACS 416 detected in the XCMS matrix and validated by targeted MS/MS; Table S3: VIP of the PLS-DA of the 417 XCMS dataset and the $\mathrm{MS}^{\mathrm{E}}$ dataset; Figure S1: Principal Component Analysis of all features detected 418 with XCMS; Figure S3: Projection to latent structure discriminant analysis modeling (PLS-DA) of all 419 features detected with XCMS; Figure S3: Projection to latent structure discriminant analysis modeling 420 (PLS-DA) of features detected by MSE; Figure S4: : Extracted ion chromatograms of DHH-MA obtained 421 by UPLC-ESI-HRMS from (A) the standard of DHH-MA and (B) urine samples. Corresponding product 422 ion spectra obtained by electrospray in the negative mode from (C) the standard of DHH-MA and (D) 423 urine samples. 
424

425

426

427

428

429

430

431

432

433

434

435

436

437

438

439

440

441

442

443

444

445

446

447

448

449

\section{REFERENCES}

(1) Lu, C.; Barr, D. B.; Pearson, M. A.; Waller, L. A. Dietary Intake and Its Contribution to Longitudinal Organophosphorus Pesticide Exposure in Urban/Suburban Children. Environ. Health Perspect. 2008, 116 (4), 537-542. https://doi.org/10.1289/ehp.10912.

(2) Pestka, J. J. Deoxynivalenol: Mechanisms of Action, Human Exposure, and Toxicological Relevance. Arch. Toxicol. 2010, 84 (9), 663-679. https://doi.org/10.1007/s00204-010-0579-8.

(3) Cascella, M.; Bimonte, S.; Barbieri, A.; Del Vecchio, V.; Caliendo, D.; Schiavone, V.; Fusco, R.; Granata, V.; Arra, C.; Cuomo, A. Dissecting the Mechanisms and Molecules Underlying the Potential Carcinogenicity of Red and Processed Meat in Colorectal Cancer (CRC): An Overview on the Current State of Knowledge. Infect. Agent. Cancer 2018, 13 (1), 3. https://doi.org/10.1186/s13027-018-0174-9.

(4) Wild, C. P. Complementing the Genome with an \&quot;Exposome\&quot;: The Outstanding Challenge of Environmental Exposure Measurement in Molecular Epidemiology. Cancer Epidemiol. Biomarkers Prev. 2005, 14 (8), 1847-1850. https://doi.org/10.1158/1055-9965.EPI05-0456.

(5) Jamin, E. L.; Bonvallot, N.; Tremblay-Franco, M.; Cravedi, J. P.; Chevrier, C.; Cordier, S.; Debrauwer, L. Untargeted Profiling of Pesticide Metabolites by LC-HRMS: An Exposomics Tool for Human Exposure Evaluation. Anal. Bioanal. Chem. 2014, 406 (4), 1149-1161. https://doi.org/10.1007/s00216-013-7136-2.

(6) Ashby, J.; Tennant, R. W. Chemical Structure, Salmonella Mutagenicity and Extent of Carcinogenicity as Indicators of Genotoxic Carcinogenesis among 222 Chemicals Tested in Rodents by the U.S. NCI/NTP. Mutat. Res. 1988, 204 (1), 17-115.

(7) Turesky, R. J. Mechanistic Evidence for Red Meat and Processed Meat Intake and Cancer Risk: A Follow-up on the International Agency for Research on Cancer Evaluation of 2015. Chim. Int. J. Chem. 2018, 72 (10), 718-724. https://doi.org/10.2533/chimia.2018.718.

(8) Hemeryck, L. Y.; Van Hecke, T.; Vossen, E.; De Smet, S.; Vanhaecke, L. DNA Adductomics to 
450

451

452

453

454

455

456

457

458

459

460

461

462

463

464

465

466

467

468

469

470

471

472

473

474

475

Study the Genotoxic Effects of Red Meat Consumption with and without Added Animal Fat in Rats. Food Chem. 2017, 230, 378-387. https://doi.org/10.1016/j.foodchem.2017.02.129.

(9) Sinha, R.; Knize, M. G.; Salmon, C. P.; Brown, E. D.; Rhodes, D.; Felton, J. S.; Levander, O. A.; Rothman, N. Heterocyclic Amine Content of Pork Products Cooked by Different Methods and to Varying Degrees of Doneness. Food Chem. Toxicol. 1998, 36 (4), 289-297.

https://doi.org/10.1016/\$0278-6915(97)00159-2.

(10) Santarelli, R.; Pierre, F.; Corpet, D. Processed Meat and Colorectal Cancer: A Review of Epidemiologic and Experimental Evidence. Nutr. Cancer 2008, 60 (2), 131-144.

https://doi.org/10.1080/01635580701684872.

(11) de La Pomélie, D.; Santé-Lhoutellier, V.; Gatellier, P. Mechanisms and Kinetics of Heme Iron Nitrosylation in an in Vitro Gastro-Intestinal Model. Food Chem. 2018, 239, 86-93. https://doi.org/10.1016/J.FOODCHEM.2017.06.092.

(12) Guéraud, F.; Taché, S.; Steghens, J.-P.; Milkovic, L.; Borovic-Sunjic, S.; Zarkovic, N.; Gaultier, E.; Naud, N.; Héliès-Toussaint, C.; Pierre, F.; et al. Dietary Polyunsaturated Fatty Acids and Heme Iron Induce Oxidative Stress Biomarkers and a Cancer Promoting Environment in the Colon of Rats. Free Radic. Biol. Med. 2015, 83, 192-200.

https://doi.org/10.1016/J.FREERADBIOMED.2015.02.023.

(13) Surya, R.; Héliès-Toussaint, C.; Martin, O. C.; Gauthier, T.; Guéraud, F.; Taché, S.; Naud, N.; Jouanin, I.; Chantelauze, C.; Durand, D.; et al. Red Meat and Colorectal Cancer: Nrf2Dependent Antioxidant Response Contributes to the Resistance of Preneoplastic Colon Cells to Fecal Water of Hemoglobin- and Beef-Fed Rats. Carcinogenesis 2016, 37 (6), 635-645. https://doi.org/10.1093/carcin/bgw035.

(14) IJssennagger, N.; Rijnierse, A.; de Wit, N.; Jonker-Termont, D.; Dekker, J.; Müller, M.; van der Meer, R. Dietary Haem Stimulates Epithelial Cell Turnover by Downregulating Feedback Inhibitors of Proliferation in Murine Colon. Gut 2012, 61 (7), 1041-1049. https://doi.org/10.1136/gutjnl-2011-300239. 


\begin{abstract}
476 (15) Carlsen, C. U.; Møller, J. K. S.; Skibsted, L. H. Heme-Iron in Lipid Oxidation. Coord. Chem. Rev. 477

(16) Sesink, A. L. A.; Termont, D. S. M. L.; Kleibeuker, J. H.; Van der Meer, R. Red Meat and Colon 479 480 Cancer: The Cytotoxic and Hyperproliferative Effects of Dietary Heme. Cancer Res. 1999, 59 (22), 5704-5709.
\end{abstract} 481 482 483 484

(18) Chevolleau, S.; Noguer-Meireles, M.-H.; Jouanin, I.; Naud, N.; Pierre, F.; Gueraud, F.; 485 486 487 488 489 490 491 492 493 494 495 496 497 498 499 500

(22) Hinchman, C. A.; Ballatori, N. Glutathione Conjugation and Conversion to Mercapturic Acids 501

(17) Bastide, N. M.; Pierre, F. H. F.; Corpet, D. E. Heme Iron from Meat and Risk of Colorectal Cancer: A Meta-Analysis and a Review of the Mechanisms Involved. Cancer Prev. Res. 2011, 4 (2), 177-184. https://doi.org/10.1158/1940-6207.CAPR-10-0113.

Debrauwer, L. Development and Validation of an Ultra High Performance Liquid Chromatography-Electrospray Tandem Mass Spectrometry Method Using Selective Derivatisation, for the Quantification of Two Reactive Aldehydes Produced by Lipid Peroxidation, HNE (4-Hydroxy-2(E)-Nonenal) and HHE (4-Hydroxy-2(E)-Hexenal) in Faecal Water. J. Chromatogr. B. Analyt. Technol. Biomed. Life Sci. 2018, 1083, 171-179. https://doi.org/10.1016/j.jchromb.2018.03.002.

(19) Guéraud, F. 4-Hydroxynonenal Metabolites and Adducts in Pre-Carcinogenic Conditions and Cancer. Free Radic. Biol. Med. 2017, 111, 196-208.

https://doi.org/10.1016/J.FREERADBIOMED.2016.12.025.

(20) Kuiper, H. C.; Miranda, C. L.; Sowell, J. D.; Stevens, J. F. Mercapturic Acid Conjugates of 4Hydroxy-2-Nonenal and 4-Oxo-2-Nonenal Metabolites Are in Vivo Markers of Oxidative Stress. J. Biol. Chem. 2008, 283 (25), 17131-17138. https://doi.org/10.1074/jbc.M802797200.

(21) Rossin, D.; Calfapietra, S.; Sottero, B.; Biasi, F. HNE and Cholesterol Oxidation Products in Colorectal Inflammation and Carcinogenesis. Free Radic. Biol. Med. 2017, 111, 186-195. https://doi.org/10.1016/J.FREERADBIOMED.2017.01.017. Can Occur as an Intrahepatic Process. J. Toxicol. Environ. Health 1994, 41 (4), 387-409. 
https://doi.org/10.1080/15287399409531852.

503 (23) Mathias, P. I.; B'Hymer, C. A Survey of Liquid Chromatographic-Mass Spectrometric Analysis of Mercapturic Acid Biomarkers in Occupational and Environmental Exposure Monitoring. J.

Chromatogr. B 2014, 964, 136-145. https://doi.org/10.1016/J.JCHROMB.2014.02.057.

(24) Scholz, K.; Dekant, W.; Völkel, W.; Pähler, A. Rapid Detection and Identification of N-Acetyl-L-

Cysteine Thioethers Using Constant Neutral Loss and Theoretical Multiple Reaction

Monitoring Combined with Enhanced Product-Ion Scans on a Linear Ion Trap Mass

Spectrometer. J. Am. Soc. Mass Spectrom. 2005, 16 (12), 1976-1984.

https://doi.org/10.1016/j.jasms.2005.08.003.

(25) Wagner, S.; Scholz, K.; Donegan, M.; Burton, L.; Wingate, J.; Völkel, W. Metabonomics and

Biomarker Discovery: LC-MS Metabolic Profiling and Constant Neutral Loss Scanning

(4), 1296-1305. https://doi.org/10.1021/ac051705s.

(26) Jian, W.; Yao, M.; Zhang, D.; Zhu, M. Rapid Detection and Characterization of in Vitro and

Urinary N -Acetyl-L-Cysteine Conjugates Using Quadrupole-Linear Ion Trap Mass Spectrometry and Polarity Switching. Chem. Res. Toxicol. 2009, 22 (7), 1246-1255.

https://doi.org/10.1021/tx900035j.

(27) Panuwet, P.; Nguyen, J. V.; Kuklenyik, P.; Udunka, S. O.; Needham, L. L.; Barr, D. B.

Quantification of Atrazine and Its Metabolites in Urine by On-Line Solid-Phase Extraction-

High-Performance Liquid Chromatography-Tandem Mass Spectrometry. Anal. Bioanal. Chem.

2008, 391 (5), 1931-1939. https://doi.org/10.1007/s00216-008-2102-0.

(28) Koszucka, A.; Nowak, A. Thermal Processing Food-Related Toxicants: A Review. Crit. Rev. Food

Sci. Nutr. 2018, 1-18. https://doi.org/10.1080/10408398.2018.1500440.

Mercapturic Acid, the Major End Metabolite of Exogenous 4-Hydroxy-2-Nonenal, Is a

Physiological Component of Rat and Human Urine. Chem. Res. Toxicol. 1998, 11 (2), 130-135. 
https://doi.org/10.1021/tx970139w.

(30) Jamin, E. L.; Bonvallot, N.; Tremblay-Franco, M.; Cravedi, J.-P.; Chevrier, C.; Cordier, S.;

Debrauwer, L. Untargeted Profiling of Pesticide Metabolites by LC-HRMS: An Exposomics Tool for Human Exposure Evaluation. Anal. Bioanal. Chem. 2014, 406 (4), 1149-1161. https://doi.org/10.1007/s00216-013-7136-2.

(31) Smith, C. A.; Want, E. J.; O'Maille, G.; Abagyan, R.; Siuzdak, G. XCMS: Processing Mass

Spectrometry Data for Metabolite Profiling Using Nonlinear Peak Alignment, Matching, and Identification. Anal. Chem. 2006, 78 (3), 779-787. https://doi.org/10.1021/ac051437y.

(32) Tautenhahn, R.; Böttcher, C.; Neumann, S. Highly Sensitive Feature Detection for High

Resolution LC/MS. BMC Bioinformatics 2008, 9 (1), 504. https://doi.org/10.1186/1471-21059-504.

Metabonomics. Anal. Chem. 2006, 78 (13), 4281-4290. https://doi.org/10.1021/ac051632c.

(35) Yang, Y.; Griffiths, W. J.; Sjövall, J.; Gustafsson, J.-Å.; Rafter, J. Liquid Chromatography-Mass

Spectrometry with Collision-Induced Dissociation of Conjugated Metabolites of

Benzol[a]Pyrene. J. Am. Soc. Mass Spectrom. 1997, 8 (1), 50-61.

https://doi.org/10.1016/S1044-0305(96)00136-5.

(36) Frigerio, G.; Mercadante, R.; Polledri, E.; Missineo, P.; Campo, L.; Fustinoni, S. An LC-MS/MS Method to Profile Urinary Mercapturic Acids, Metabolites of Electrophilic Intermediates of Occupational and Environmental Toxicants. J. Chromatogr. B 2019, 1117, 66-76. https://doi.org/10.1016/J.JCHROMB.2019.04.015. 
554 (37) Plumb, R. S.; Johnson, K. A.; Rainville, P.; Smith, B. W.; Wilson, I. D.; Castro-Perez, J. M.;

555

556

557

558

559

560

561

562

563

564

565

566

567

568

569

570

571

572

573

574

575

576

577

578

579
Nicholson, J. K. UPLC/MSE; a New Approach for Generating Molecular Fragment Information for Biomarker Structure Elucidation. Rapid Commun. Mass Spectrom. 2006, 20 (13), 19891994. https://doi.org/10.1002/rcm.2550.

(38) Alary, J.; Fernandez, Y.; Debrauwer, L.; Perdu, E.; Guéraud, F. Identification of Intermediate Pathways of 4-Hydroxynonenal Metabolism in the Rat. Chem. Res. Toxicol. 2003, 16 (3), 320327. https://doi.org/10.1021/tx025671k.

(39) Alary, J.; Debrauwer, L.; Fernandez, Y.; Paris, A.; Cravedi, J.-P.; Dolo, L.; Rao, D.; Bories, G. Identification of Novel Urinary Metabolites of the Lipid Peroxidation Product 4-Hydroxy-2Nonenal in Rats. Chem. Res. Toxicol. 1998, 11 (11), 1368-1376.

https://doi.org/10.1021/tx980068g.

(40) Sumner, L. W.; Amberg, A.; Barrett, D.; Beale, M. H.; Beger, R.; Daykin, C. A.; Fan, T. W.-M.; Fiehn, O.; Goodacre, R.; Griffin, J. L.; et al. Proposed Minimum Reporting Standards for Chemical Analysis Chemical Analysis Working Group (CAWG) Metabolomics Standards Initiative (MSI). Metabolomics 2007, 3 (3), 211-221. https://doi.org/10.1007/s11306-0070082-2.

(41) Winter, C. K.; Segall, H. J.; Jones, A. D. Distribution of Trans-4-Hydroxy-2-Hexenal and Tandem Mass Spectrometric Detection of Its Urinary Mercapturic Acid in the Rat. Drug Metab. Dispos. $15(5), 608-612$.

(42) Bastide, N. M.; Chenni, F.; Audebert, M.; Santarelli, R. L.; Tache, S.; Naud, N.; Baradat, M.; Jouanin, I.; Surya, R.; Hobbs, D. A.; et al. A Central Role for Heme Iron in Colon Carcinogenesis Associated with Red Meat Intake. Cancer Res. 2015, 75 (5), 870-879. https://doi.org/10.1158/0008-5472.CAN-14-2554.

(43) Guo, J.; Villalta, P. W.; Turesky, R. J. Data-Independent Mass Spectrometry Approach for Screening and Identification of DNA Adducts. Anal. Chem. 2017, 89 (21), 11728-11736. https://doi.org/10.1021/acs.analchem.7b03208. 
580 (44) Contrepois, K.; Jiang, L.; Snyder, M. Optimized Analytical Procedures for the Untargeted 581 Metabolomic Profiling of Human Urine and Plasma by Combining Hydrophilic Interaction (HILIC) and Reverse-Phase Liquid Chromatography (RPLC)-Mass Spectrometry. Mol. Cell. Proteomics 2015, 14 (6), 1684-1695. https://doi.org/10.1074/mcp.M114.046508. 